\title{
Graphene Oxide/Silver Nanocomposites as Antifouling Coating on Sensor Housing Materials
}

\author{
Xiaoxue Zhang ${ }^{1} \cdot \varnothing y v i n d$ Mikkelsen ${ }^{1}$
}

Received: 18 May 2020 / Accepted: 24 November 2020/Published online: 28 January 2021

(C) The Author(s) 2021

\begin{abstract}
These days, sensors are widely used in a variety of underwater sites like marine monitoring, fish-farming and water quality monitoring. However, to achieve reliable sensor data from long-term monitoring in aqueous solution, several challenges still need to be solved. Biofilm formation both on sensor housings and membranes is among one of the most serious challenges, which strongly influences the sensor responds and the validity of the results. To prevent biofilm growth, a series of graphene oxide (GO)/silver nanoparticles (Ag NPs) nanocomposites (GOA) have been developed and coated on sensor housing materials, e.g. polypropylene. The antifouling property of the GOA nanocomposite has been demonstrated by antifouling tests using Halomonas. Pacifica (Baumann et al.) Dobson and Franzmann (ATCC® 27122) (H. Pacifica) and a mixture of marine algae. The antifouling property of GOA composites has been proved to be closely related to the dispersibility of Ag NP. The overall work might provide valuable insight into developing antifouling materials for sensors in general.
\end{abstract}

Keywords Graphene oxide · Silver nanoparticles · Nanocomposites · Antifouling

\section{Introduction}

Nowadays, marine monitoring stations haven been set up worldwide in order to achieve information about seawater quality, marine ecosystems and environmental contaminants [1-3]. In these systems, in-situ sensors have been widely used to acquire online, constantly and real-time data and thus play a vital role in marine monitoring $[4,5]$. However, surface biofouling on sensors housings and support structures could cause inaccurate measurement and shorten sensor's lifetime [4, 6]. Therefore, in order to increase the validity of sensor data, it is urgent to find suitable anti-fouling materials to inhibit or minimize the growth of biofilm. Antifouling coating have been considered as an effective approach to protect sensors from fouling.

To date, tributyltin (TBT) self-polishing paints have been among the most efficient antifouling coatings [7-9]. However, tributyltin (TBT) based coating has been banned

Xiaoxue Zhang

xiaoxue206258@gmail.com

1 Department of Chemistry, NTNU, 7491 Trondheim, Norway since 2008 due to its toxic nature and non-target organisms. In addition, the use of biocidal paints such as copper paints may also pose problems for environment [10]. Hence, more attention has been attracted to develop alternative materials like superhydrophobic and nanostructured fouling release (FR) coatings. Sherif A. EI-Safty has made a series of silicon-based FR released coatings, with graphene oxide sheet-alumina nanorod, titanium dioxide-silicone dioxide nanorod and $\beta-\mathrm{MnO}_{2}$ nanorod working as nanofillers. The well dispersion of nanofillers and nanostructured surface lead to superhydrophobic and self-cleaning property, which in turn leading to outstanding antifouling property. Thus, nanoscale materials play a promising role in developing antifouling coatings [11-14].

Antifouling property of metal and metal oxide nanoparticles $\left(\mathrm{Ag}, \mathrm{Cu}, \mathrm{ZnO}, \mathrm{TiO}_{2}\right.$ ) have been well documented by a number of studies [15-17], and silver nanoparticles (Ag NPs) were found to be the most effective one after testing 17 different kinds of metal compounds on Escherichia coli JM 109 [18]. Meanwhile, Ag NPs have been used in numerous anti-bacterial applications due to their efficiency to prevent growth of a broad-spectrum of microorganisms while being low in cytotoxicity to higher 
animals [19-21]. Moreover, effective inhibiting of biofilm formation has been achieved in various fields by coating with Ag NPs like catheters, titanium implants, pipeline and membrane [22-24]. However, aggregation issues caused by high surface energy and extremely large surface area, difficulties to immobilize on surfaces, as well as burst release of silver ions, throw huge challenges for antibacterial surface coating of Ag NPs. Thus, immobilizing Ag NPs on support matrix like polymers, zeolite frameworks and graphene oxide (GO) make a breakthrough in solving those problems.

GO, a single-atom thick carbon sheet with abundant of oxygen functional groups, has attracted tremendous attention due to its unique nanostructure [25-27]. The oxygen groups could serve as the nucleation sites for the growth and anchoring of metals and metallic compounds to obtain GO/nanoparticle composites. This could improve the particles dispersibility and strengthen the contact between nanoparticles and bacteria, resulting in the enhanced antibacterial property [28-32]. Moreover, the excellent antifouling efficiency, chemical resistance (acid/base/salt) and being eco-friendly make GO a popular choice as protective surface-coatings. For instance, GO nanopaints developed by Karthikeyan et al. have efficiently inhibited biofilm growth in sea water [33]. Sirus et al. used GO nanosheets to improve antifouling property of polyethersulfone membrane (PES) membranes [34].

Here, GOA nanocomposites have been successfully prepared by in-situ reducing of $\mathrm{AgNO}_{3}$ solution on the surface of $\mathrm{GO}$ with $\mathrm{NaBH}_{4}$. It is worth highlight that the reaction doesn't require a vacuum or inert atmosphere. Owning to its excellent antifouling property, GOA nanocomposites have wide application potentials in e.g. wastewater treatment, ultrafiltration and marine surfaces [35-37]. To the best of our knowledge, application and optimalization of GOA for sensor housing materials for use in marine environment and especially in aquaculture relevant solutions are lacking in literature. In the current work, we have studied the surface property of GOA coating layer, which proved good dispersion of Ag NPs can lead to a homogenous surface with high WCA and low surface free energy (SFE). We also proved the antifouling property of GOA nanocomposite against a common marine bacteria $H$. Pacifica and a unique mix of six marine microalgae. Above all, the GOA composite designed in this work could be a promising potetial coating on sensor in future.

\section{Materials and Methods}

\section{Chemicals}

Graphene oxide was purchased from Abalonyx Norway. Silver nitrate $\left(\mathrm{AgNO}_{3},>99.0 \%\right)$, sodium borohydride $\left(\mathrm{NaBH}_{4}\right), 1 \%$ crystal violet solution $\left(\mathrm{C}_{25} \mathrm{H}_{30} \mathrm{C}_{1} \mathrm{~N}_{3}\right)$, glycerol, dimethyl sulfoxide (DMF, 99.7\%), polyvinylidene difluoride (PVDF) and N-methyl pyrrolidinone (NMP, 99.5\%) were purchased from Sigma-aldrich, Norway. $H$. Pacifica (Baumann et al.) Dobson and Franzmann (ATCC ${ }^{\circledR}$ 27122) was purchased from the American Type Culture Collection (ATCC). Zobell Marine Broth 2216 was purchased from HiMedia Laboratories, India. Shellfish diet $1800^{@}$ was purchased from Reed Mariculture, Norway. Adam medium was provided by Department of Biology at Norwegian University of Science and Technology (NTNU). SYTO 9 was purchased from Thermofisher Scientific, Norway. All chemicals are used without purification.

\section{Preparation of GOA Nanocomposite}

GOA nanocomposite was prepared by an in-situ method as illustrated in Scheme 1. In brief, $125 \mathrm{mg}$ GO powders were dispersed in $437.5 \mathrm{ml}$ deionized water (DI) under $10 \mathrm{~min}$ of probe sonication. Subsequently, different amount of $10^{-2}$ $\mathrm{M} \mathrm{AgNO}_{3}$ was added into GO suspension followed by $30 \mathrm{~min}$ of ultrasonic treatment. The sonicated dispersion was then kept in ice bath for $30 \mathrm{~min}$. Then $\mathrm{NaBH}_{4}$ was dropwise added into the cooled mixture, and the reaction was kept still in room temperature for $12 \mathrm{~h}$. The solid precipitation was collected after treatments of centrifugation and washing (with DI water and $100 \%$ ethanol in turn) for several times. Finally, the resulting GOA nanocomposites were dried in a vacuum oven at $60{ }^{\circ} \mathrm{C}$ for $24 \mathrm{~h}$. In this work, GOA nanocomposites were named based on the mass ratio of $\mathrm{GO}$ to $\mathrm{AgNO}_{3}$. For example, GOA10 represents the mass ratio of $\mathrm{GO}$ to $\mathrm{AgNO}_{3}$ is 10 .

\section{Characterization of GO and GO Nanocomposites}

\section{Structure and Morphology Characterization}

XRD measurements were carried out in DaVincil. UVvisible absorption spectra were recorded by Cary 60 UVvis at the range of 200 to $700 \mathrm{~nm}$. Raman spectra were recorded by InVia Reflex Spectrometer System with $532 \mathrm{~nm}$ laser excitation. The mass loss and weight percentage of $\mathrm{Ag}$ NPs were characterized with a 


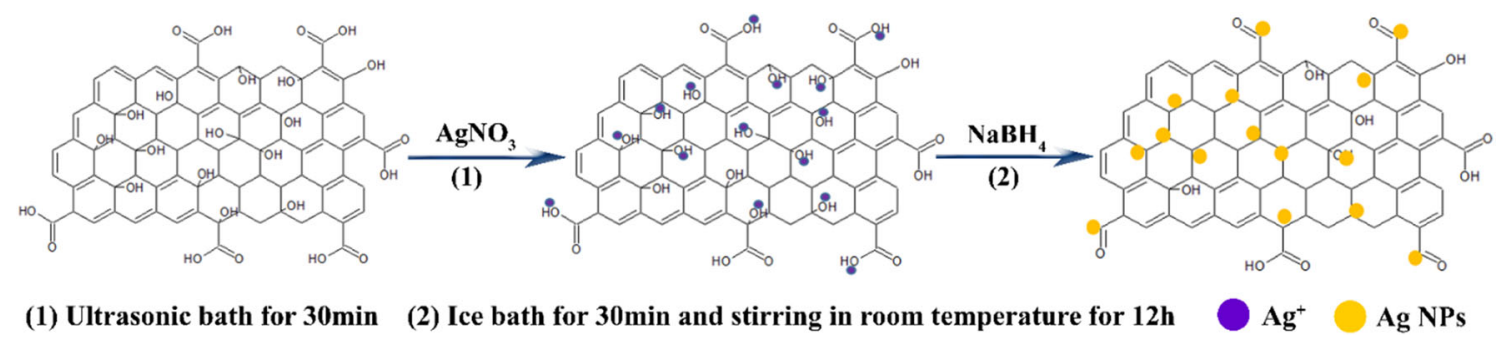

Scheme 1 Illustration depicting preparation of GOA nanocomposite

thermogravimetric analyser (TG Hugin). The measurement was obtained with $10 \mathrm{mg}$ of dried samples at a heating rate of $10{ }^{\circ} \mathrm{C} / \mathrm{min}$ under a synthetic air flow rate of $100 \mathrm{ml} / \mathrm{min}$. And the temperature was increased from $100{ }^{\circ} \mathrm{C}$ to $800{ }^{\circ} \mathrm{C}$. TEM images of GO and GOA nanocomposites were obtained by using an in-lens cold field emission electron microscopy (Hitachi S-5500 S(T)EM) with an accelerating voltage at $30 \mathrm{KV}$.

\section{Surface Property Measurement}

The surface property of the synthesized materials was analyzed with Atomic force Microscopy (AFM, Veeco Metrology). Surface morphology and roughness were measured under PeakForce Quantitative NanoMechanics. Static WCA was recorded with drop shape analyzer DSA25 (Krüss, Germany) by sessile drop technique. Five contact angle measurements were performed with deionized water for each sample. To determine SFE of GO and GOA nanocomposite, surface contact angles of pure water (polar liquid) and diiodomethane (disperse liquid) were measured. Owens, Wendt, Rabel and Kaelble (OWRK model) [38, 39] was applied to calculate the SFE of GO and GOA. The equilibrium contact angle is well defined by Yong's equation [39].

$\sigma_{s}=\sigma_{s l}+\sigma_{l} \cos \theta$

where $\sigma_{s}, \sigma_{l}$ and $\sigma_{s l}$ represent the interfacial free energies at solid-vapor, liquid-vapor and solid-liquid respectively. $\theta$ is the contact angle of liquid on a solid surface.

OWRK model is based on the assumption that the interfacial energy can be split up between the molecules according to the forces of interaction. In doing so, the surface energy is divided into polar part and disperse part. Thus, the interfacial free energy for $\sigma_{s}$ and $\sigma_{l}$ can be illustrated in Eqs. (2) and (3):

$\sigma_{l}=\sigma_{l}^{d}+\sigma_{l}^{p}$

$\sigma_{s}=\sigma_{s}^{d}+\sigma_{s}^{l}$

where $\boldsymbol{\sigma}_{s}^{\boldsymbol{d}}$ and $\boldsymbol{\sigma}_{s}^{p}$ represent the dispersive and polar surface free energy of solid, $\sigma_{l}^{d}$ and $\sigma_{l}^{p}$ represent the dispersive and polar surface free energy of liquid, respectively. $\sigma_{s l}$ can be obtained based on geometric method as the following equation:

$\sigma_{s l}=\sigma_{s}+\sigma_{l}-2\left(\sqrt{\sigma_{s}^{d} \sigma_{l}^{d}}+\sqrt{\sigma_{s}^{p} \sigma_{l}^{p}}\right)$

substituting Eq. (4) in Eq. (1), the following expression can be obtained,

$\sigma_{l}(1+\cos \theta)=2\left(\sqrt{\sigma_{s}^{d} \sigma_{l}^{d}}+\sqrt{\sigma_{s}^{p} \sigma_{l}^{p}}\right)$

Once contact angles of polar and disperse liquid on GO and GOA nanocomposites are known, $\boldsymbol{\sigma}_{s}^{\boldsymbol{d}}$ and $\boldsymbol{\sigma}_{\boldsymbol{s}}^{p}$ can be calculated from Eq. (5). Therefore, $\sigma_{s}$ can be obtained from Eq. (3).

\section{Anti-microfouling Activity Against $H$. pacifica}

H. Pacifica, a gram-negative bacterium was used to evaluate antifouling property of GOA nanocomposite, which has been described by Maxine Swee-Li Yee [35]. In short, H. Pacifica was cultured overnight in marine broth stationarily at $26{ }^{\circ} \mathrm{C}$. Then it was seeded into 96-well plate at an optical density $600 \mathrm{~nm}$ of 0.01 with or without $1 \mathrm{mg} / \mathrm{ml}$ of GOA nanocomposite. Subsequently, the 96-well plates with overnight culture were incubated at $26{ }^{\circ} \mathrm{C}$ under stationary condition for $24 \mathrm{~h}$. The plates were washed with water two times after discarding medium inside. Then $0.1 \%$ aqueous solution of crystal violet was added into each well to stain bacteria on the biofilm for $30 \mathrm{~min}$ at room temperature. The stained bacteria in each well was washed with water twice, following being solubilized with $30 \%$ acetic acid for $15 \mathrm{~min}$. Finally, the solubilized stain was transferred into a new 96-well plates, with quantifying biofilm inhibition by Tecan infinite M200 PRO, in order to measure the optical density at $570 \mathrm{~nm}\left(\mathrm{OD}_{570}\right)$.

\section{Algal Adhesion Evaluation}

Shellfish diet $1800^{@}$ which consists of 6 marine microalgae was used to evaluate algal adhesion property. GOA1 which has been proved to be the best antifouling property against H. Pacifica, was coated on the surface on PP substrate by 
drop coating method. In brief, $90 \mathrm{wt} \%$ GOA 1 and $10 \mathrm{wt} \%$ of PVDF were mixed in the presence of NMP. The slurry was made by ball milling followed by drop casting onto PP substrates. The same method was used to prepare GO coating layer. After air drying the coated PP at room temperature for $10 \mathrm{~h}$, both coated and uncoated PP substrates were immersed into Adam medium for one week, with adding quantitative algae every other day. After rinsing out the loosen biofilm on the surface of PP substrates, all samples were stained with $10 \mu \mathrm{M}$ solution of SYTO9 for $15 \mathrm{~min}$. Then confocal laser scanning microscopy (CLSM, Lecia SP5) was used to characterize biofilm formation on both coated and bare PP substrates.

\section{Results and Discussion}

\section{Synthesis of GOA Nanocomposites}

A simple method was used to synthesize GOA nanocomposites through in-situ reduction of $\mathrm{AgNO}_{3}$ on the surface of $\mathrm{GO}$ without using vacuum and inert gas. In comparison with pristine GO, GOA nanocomposites benefit from both components from the system. The plentiful hydroxyl groups and wrinkled surface of GO provide abundant nucleation sites and large surface for Ag NPs to attach. The antifouling property is attribute to the presence of Ag NPs, whereas GO provide high surface for cellular interaction and deposition. Additionally, the distribution of Ag NPs was directly affected by the content of Ag NPs, which has close influence on antifouling property.

\section{Characterization of GO and GOA Nanocomposites}

UV measurements is one of the most powerful and informative techniques to investigate the formation of Ag NPs on GOA nanocomposites. As shown in Fig. 1, the UV spectrum of GO clearly shows the characteristic peaks at $230 \mathrm{~nm}$ and $300 \mathrm{~nm}$ that caused by the $\pi-\pi^{*}$ transitions of the $\mathrm{C}-\mathrm{C}$ bonds and the $\mathrm{n}-\pi^{*}$ transitions of $\mathrm{C}=\mathrm{O}$ bonds, respectively $[40,41]$. In the UV spectra of GOA nanocomposites, a new peak at $\sim 400 \mathrm{~nm}$ is associated with the surface plasmon resonance (SPR) absorption band of $\mathrm{Ag}$ NPs, indicating the formation of $\mathrm{Ag}$ NPs [42]. Meanwhile, the intensity of SPR is directly related with the amount of Ag NPs, the intensity becomes stronger with the increased Ag NPs. Also, with the increased content of Ag NPs, the shoulder at $300 \mathrm{~nm}$ disappears gradually and the absorption peak at $230 \mathrm{~nm}$ gradually shifts to $264 \mathrm{~nm}$. This indicates the extensive conjugated $\mathrm{sp}^{2}$-carbon network is restored due to the reduction of $\mathrm{GO}$ by $\mathrm{NaBH}_{4}$.

The functionalization of GO with Ag NPs was further checked by Raman Spectroscopy. Fig. 2 performs a

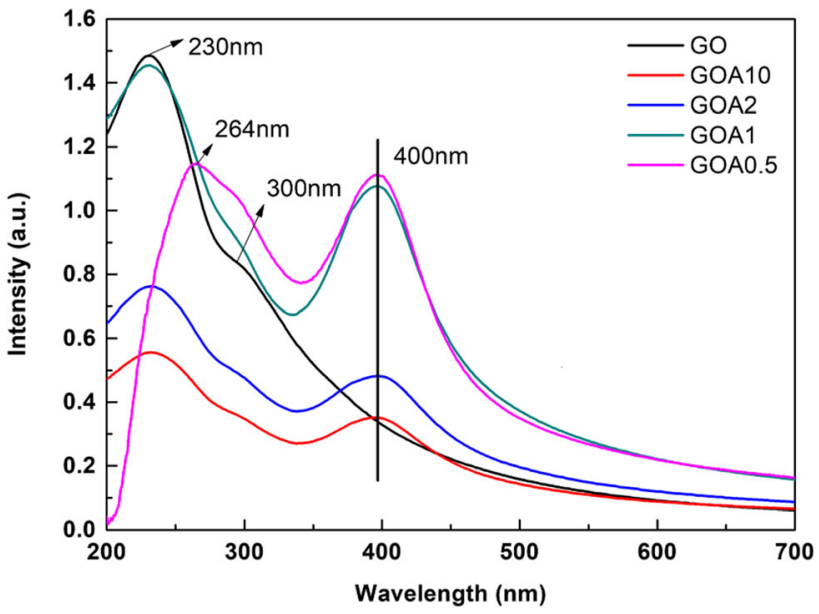

Fig. 1 UV-visible spectra of GO and GOA nanocomposites with different content of Ag NPs

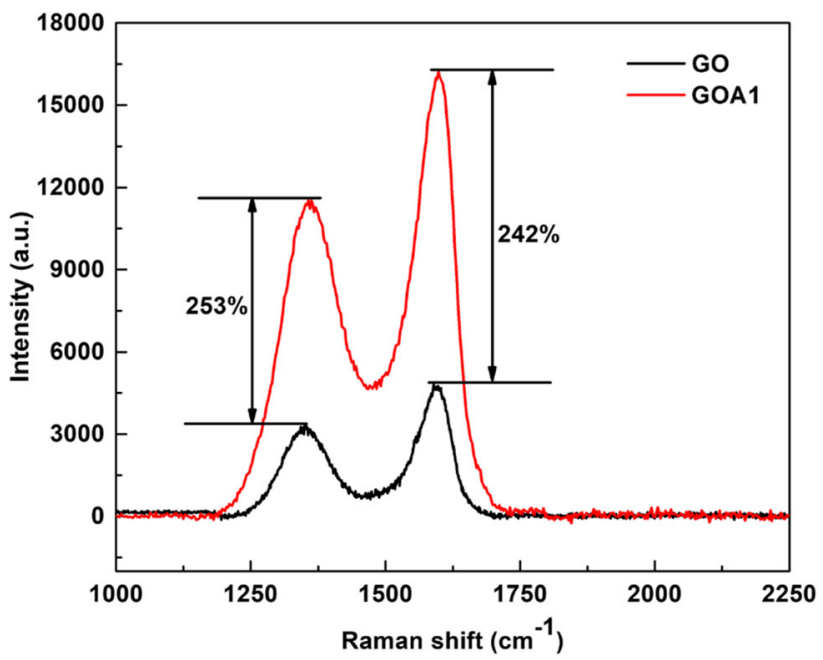

Fig. 2 Raman spectra of GO and GOA1 nanocomposites

comparison of Raman spectra between GO and GOA1. GO displayed two dominant peaks at $\sim 1361 \mathrm{~cm}^{-1}$ (D band) and $\sim 1594 \mathrm{~cm}^{-1}$ (G band). The D band is related to the defects associated with vacancies and edge defects while the $\mathrm{G}$ band is assigned to the $\mathrm{E}_{2 \mathrm{~g}}$ mode of $\mathrm{SP}^{2}$ carbon atoms. Compared to GO, GOA1 exhibits up-shifted D band (from 1351 to $1360 \mathrm{~cm}^{-1}$ ), the up-shifted $\mathrm{G}$ band (from 1549 to $1599 \mathrm{~cm}^{-1}$ ) and the increased $\mathrm{I}_{\mathrm{D}} / \mathrm{I}_{\mathrm{G}}$ ratio (from 0.68 to 0.71 ). These results indicate that the chemical reduction reduces the $\mathrm{SP}^{2}$ domains and increases the degree of disorder of GO during the preparation of GOA [41]. In addition, the peak intensity of D and G band of GOA1 increased by $253 \%$ and $242 \%$, respectively, compared with those of GO. This is attributed to the surface-enhanced Raman scattering (SERS) caused by the formation of charge-transfer complexes during the interaction between Ag NPs and GO nanosheets [25]. 
The formation of GOA nanocomposites was investigated by XRD (Fig. 3). The peak of GOA1 at around $11.26^{\circ}$ disappears compared with GO. This is because the anchoring of Ag NPs on the surface of GO prevents the stacking of the GO layers. Besides, four new peaks at around $38.26^{\circ}, 44.42^{\circ}, 64.63^{\circ}$ and $77.54^{\circ}$ in the XRD pattern of GOA1 are indexed to the (111), (200), (220) and (311) plane of cubic Ag crystal, respectively.

Thermogravimetric analysis of GO and GOA nanocomposites was investigated by TGA (Fig. 4). GO exhibits two main stages of weight loss. The first region weight loss (from 150 to $215^{\circ} \mathrm{C}$ ) is attributed to the decomposition of oxygenated functional groups on $\mathrm{GO}$ surface, whereas the second weight loss (from $535{ }^{\circ} \mathrm{C}$ to $670^{\circ} \mathrm{C}$ ) is assigned to the decomposition of graphitic layer [43]. GOA nanocomposites show a significant reduction in decomposition temperature, especially for the second weight loss, which is at $\sim 400{ }^{\circ} \mathrm{C}$. This is associated with the presence of Ag NPs acting as catalysts, thus reducing the decomposition temperature of GOA nanocomposites. TGA was also applied to estimate the amount of Ag NPs by analyzing the weight loss at the temperature above $670{ }^{\circ} \mathrm{C}$. According to TGA curves, the relative percentage of $\mathrm{Ag}$ NPs in GOA nanocomposites is about $16 \mathrm{wt} \%$ (GOA2), 22 $\mathrm{wt} \%$ (GOA1) and $70 \mathrm{wt} \%$ (GOA0.5), respectively.

The typical morphology features of GO and GOA nanocomposites were investigated through TEM as shown in Fig. 5. GO displays a wrinkled and folded structure which can provide large surface for Ag NPs to attach. TEM micrographs of GOA confirm the Ag NPs are successfully attached on GO. In Fig. 5b-d, the Ag NPs are homogenously distributed on the surface of GO nanosheets with size distribution at around 10-15 nm. However, the dispersibility of Ag NPs becomes poor with the increased amount of Ag NPs after GOA1, and serious particles aggregation was observed in GOA0.5 (see Fig. 5e).

Furthermore, the EDX spectrum of GOA1 (Fig. 5e) exhibits the characteristic peaks of $\mathrm{C}, \mathrm{O}$ and $\mathrm{Ag}$, indicating

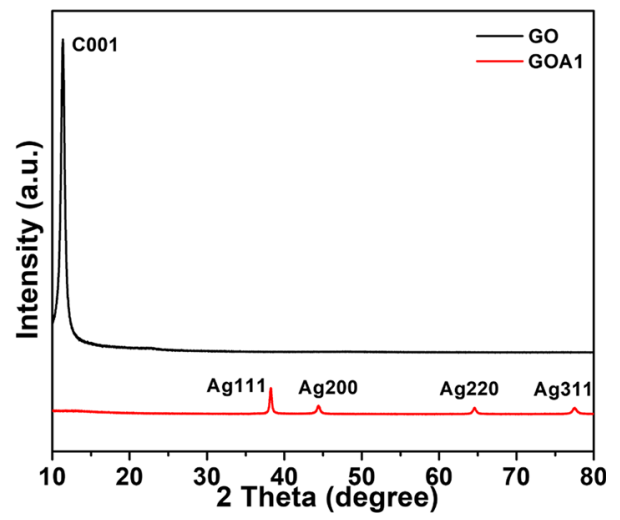

Fig. 3 X-ray spectra of GO and GOA1 nanocomposites

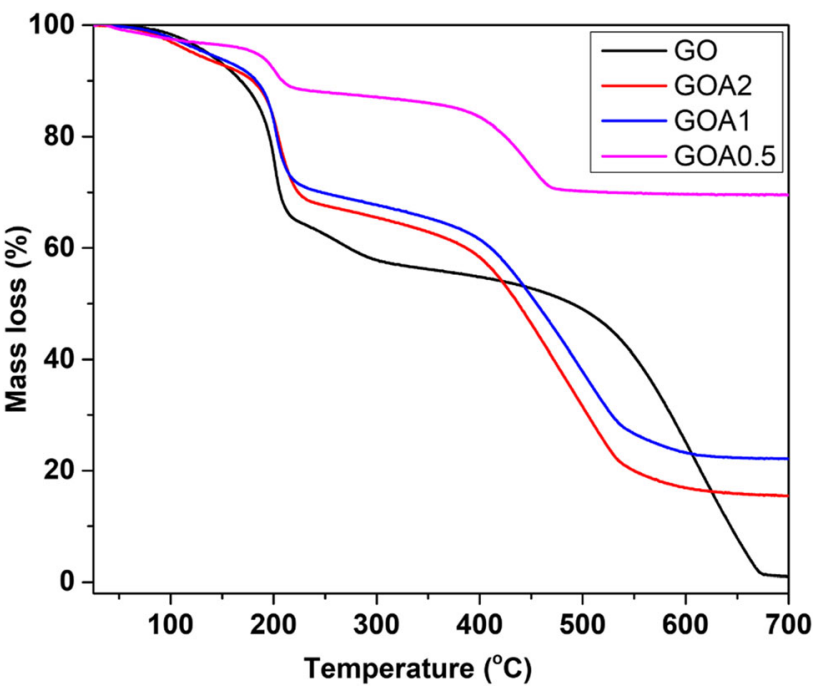

Fig. 4 TGA curves for GO and GOA nanocomposites with different content of Ag NPs

the successful preparation of GOA nanocomposites. This is in accordance with the results of UV, XRD, Raman spectra and TGA curves above. By the way, the observed $\mathrm{Si}$ and $\mathrm{Cu}$ peaks are due to the sample substrate and $\mathrm{Cu}$ grid where the sample is prepared.

\section{Surface property of GO and GOA nanocomposites}

Topography of GO and GOA nanocomposites was investigated through AFM, as shown in Fig. 6. There's no significant difference observed about GO and GOA nanocomposites' topography. However, the surface roughness $(\mathrm{Ra})$ displays obvious differences among GO and GOA nanocomposites, as shown Fig. 7. GO shows a $\mathrm{Ra}$ of $38.6 \mathrm{~nm}$, while the increased content of Ag NPs until $22 \mathrm{wt} \%$ (GOA1) raises $\mathrm{Ra}$ to $59.0 \mathrm{~nm}$ due to the homogenous distribution of Ag NPs. On the other hand, the increased content of Ag NPs from $22 \mathrm{wt} \%$ (GOA1) to $70 \mathrm{wt} \%$ (GOA0.5) reduces $\mathrm{Ra}$ to $44.6 \mathrm{~nm}$ due to aggregation of Ag NPs. In addition, with good dispersibility of Ag NPs, the raised content of Ag NPs results in high WCA, whereas the cluster of Ag NPs decreases WCA. Conversely, the raised content of Ag NPs until $22 \mathrm{wt} \%$ (GOA1) reduces the $\boldsymbol{\sigma}_{\boldsymbol{s}}$ to $37.62 \mathrm{mN} / \mathrm{m}$, while the increased amount of Ag NPs from 22 to $70 \mathrm{wt} \%$ raises $\sigma_{\boldsymbol{s}}$ to $42.36 \mathrm{mN} / \mathrm{m}$ due to the aggregation of Ag NPs. In conclusion, with good distribution, raised content of Ag NPs induces high Ra, high WCA and low SFE which benefit for antifouling property. On the other hand, the cluster of Ag NPs has negative impact on antifouling property due to low $\mathrm{Ra}$, low WCA and high SFE. 
A

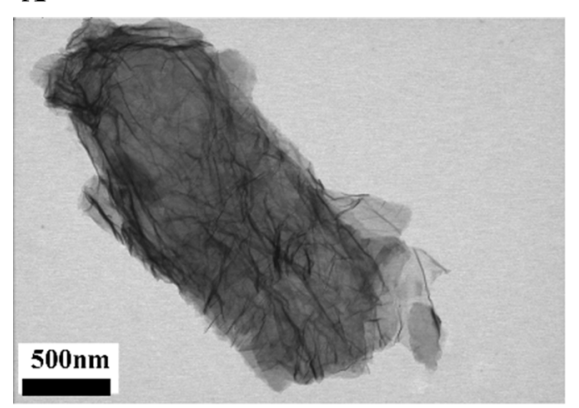

D

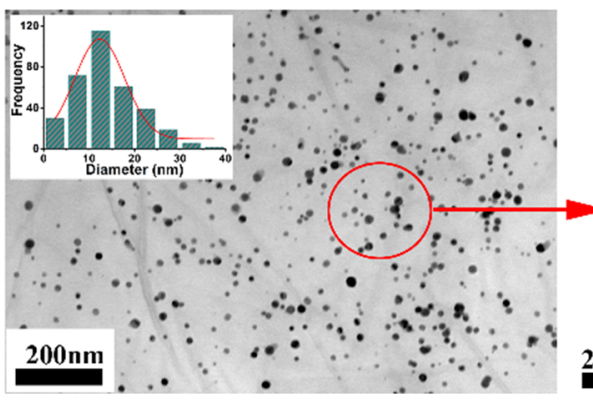

B

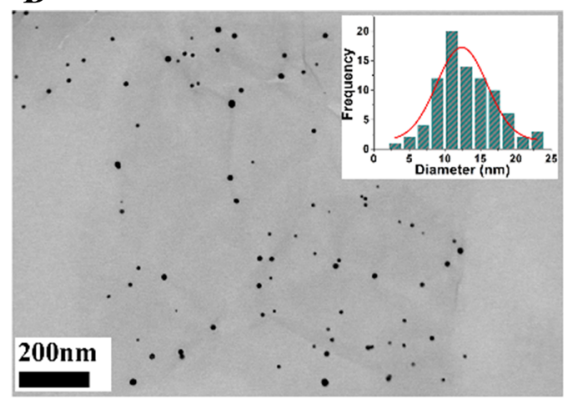

$\mathbf{E}$

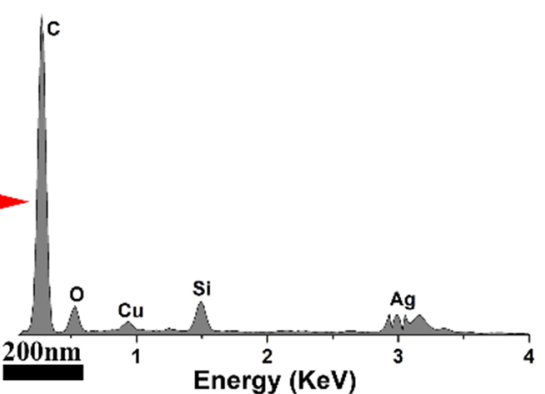

C
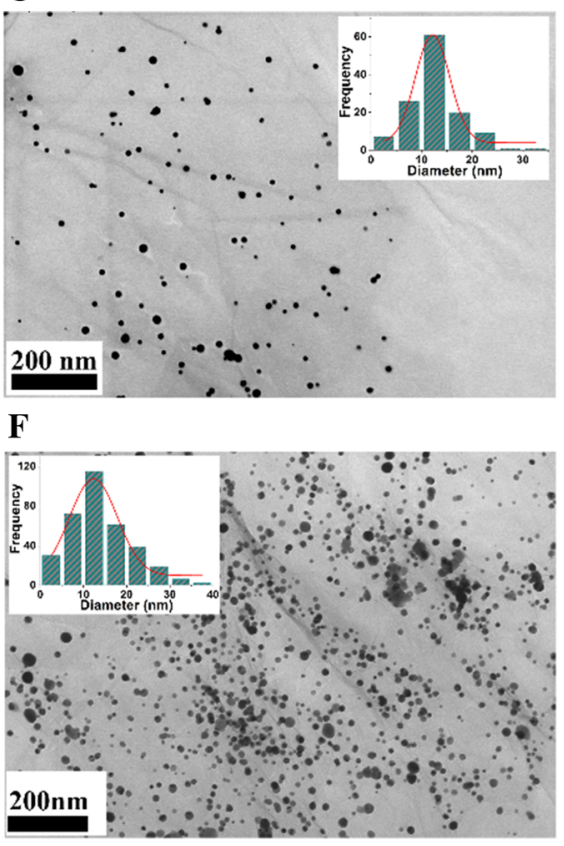

Fig. 5 TEM images of GO and GOA nanocomposites a GO, b GOA10, $\mathbf{c}$ GOA2, $\mathbf{d}$ GOA1, e EDX spectrum of GOA1 and $\mathbf{f}$ GOA0.5; Insert graphs represent size distribution of Ag NPs of GOA nanocomposites
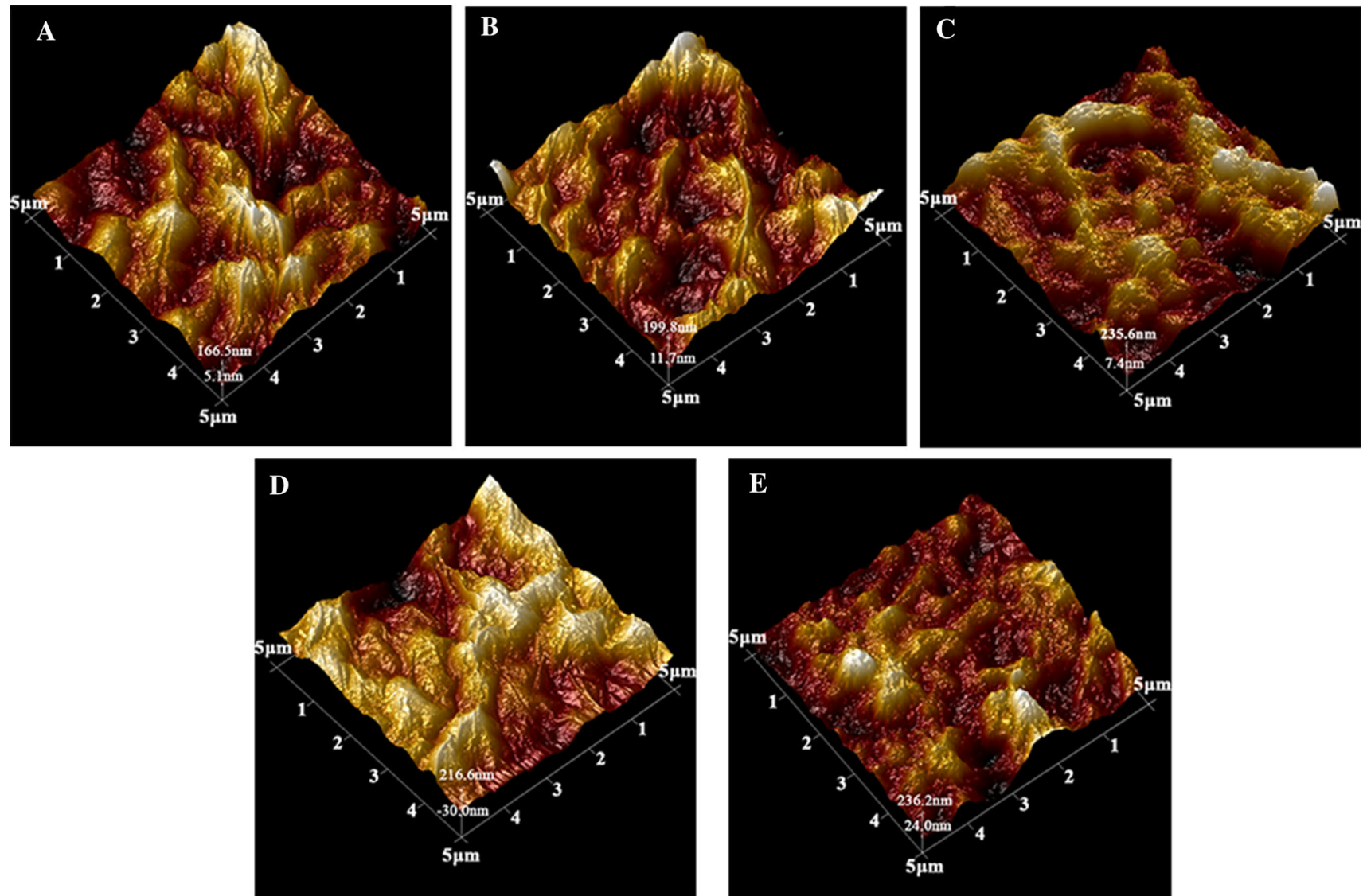

Fig. 6 Three-dimensional AFM images of GO and GO nanocomposites a GO, b GOA10, c GOA2, d GOA1 and e GOA0.5 
Fig. 7 Comparison of WCA, SFE and Ra of GO and GOA nanocomposites

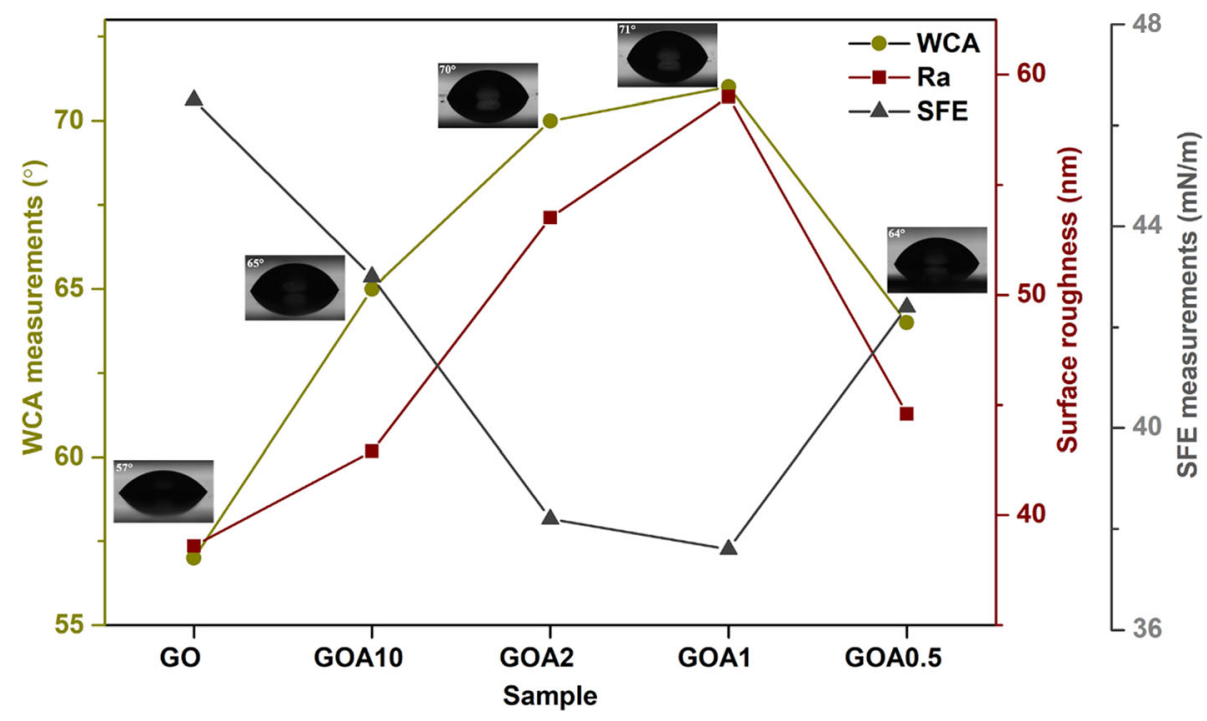

\section{Antifouling Property Against $\boldsymbol{H}$. Pacifica}

The biofilm inhibition assay was conducted with marine bacteria $H$. Pacifica. As shown in Fig. 8, biofilm formation is inhibited dramatically after treating with GOA nanocomposites. GO shows almost no antifouling property, while GOA nanocomposites present more than $80 \%$ average biofilm inhibition against $H$. Pacifica. Even GO has no potent antifouling property, it serves as an effective platform for Ag NPs to attach on and strengthens the contact between Ag NPs and H. Pacifica, which contributes to antifouling efficiency [31]. Moreover, the antifouling performance of GOA is attributed to its homogenous surface with high WCA and low SFE caused by well-dispersion of Ag NPs. Among the synthesized GOA, GOA1 exhibits the best antifouling performance due to its homogenous surface (the highest WCA with the value $71^{\circ}$ and the lowest $\mathrm{SFE}$ with the value of $37.62 \mathrm{mN} / \mathrm{m}$ ) resulting from welldispersion of $\mathrm{Ag}$ NPs. On the other hand, the increased amount of Ag NPs from GOA1 (22 wt\%) to GOA0.5 (70 wt $\%$ ) induces aggregation of Ag NPs. This has negative influence on antifouling property. Above all, antifouling property of GOA nanocomposites is closely related to homogeneous distribution of Ag NPs.

\section{Antifouling Property Against Algae}

GOA1 was selected for antifouling test against mixture of algae, because it presents the highest loading of Ag NPs with good distribution. The Anti-fouling effect of GOA1 on algae was examined by immersing bare PP,GO coated and GOA1 coated PP substrates in Adam medium with continuous addition of algae for 1 week. As shown in Fig. 9Aa, both bare PP and GO coated PP surface exhibit obvious green algae biofilm; however, there is no obvious fouling on GOA1 coated surface. Biofilm on bare PP, GO and GOA1 coated PP surface were further studied by CLSM. CLSM is a useful instrument to characterize microfouling, which gives data about the density and thickness of biofilm. As shown in Fig 9B, algae cells are
Fig. 8 A Biofilm staining with crystal violet and average OD of control sample, GO, GOA nanocomposites at $0.1 \mathrm{mg} / \mathrm{ml}$; B Quantification of antifouling activity of control sample, GO, GOA nanocomposites. Bars represent mean $\pm \mathrm{SD}$ of three independent experiments. $(P<0.05$, student's $t$-test $)$
A

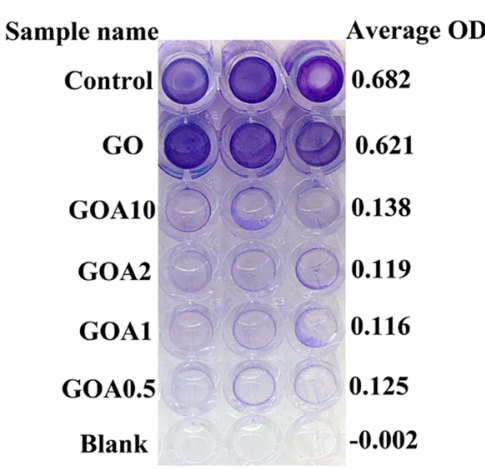

B

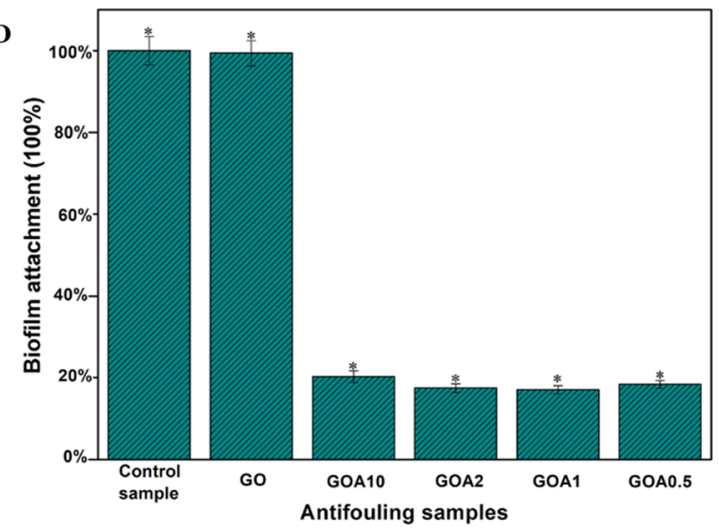


Fig. 9 A Optical images of bare PP (a), GO coated PP (b) and GOA1 coated PP (c) after exposure in algae solution for 1 week; Fig. 8. B CLSM images of bare PP (a), GO coated PP (b) and GOA1 coated PP (c) after exposure in algae solution for 1 week

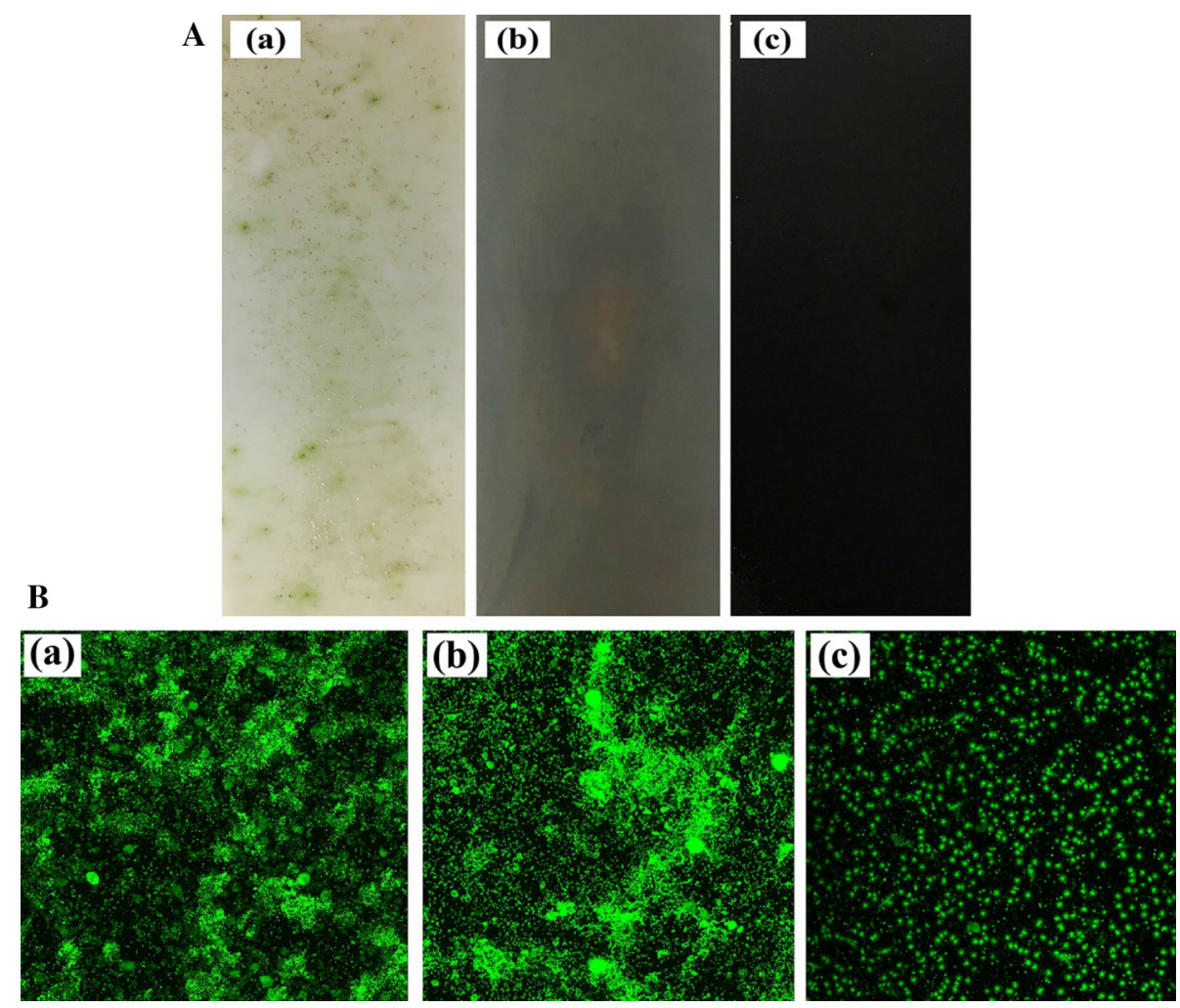

observed across bare PP, GO and GOA coated PP surfaces; however, the density of cells on bare PP and GO coated PP surface is observed to be thicker and denser compare with the GOA1 coated PP surface. The biofilm thickness on bare PP surface, GO and GOA1 coated PP surface is about 46, 42 and $20 \mu \mathrm{m}$, respectively. The results indicate that the good distribution of Ag NPs of GOA1 leading to homogenous surface, low SFE and high WCA which in turn results in good antifouling property.

\section{Conclusions}

In summary, a series of GOA nanocomposite with different amount of Ag NPs were synthesized through in-situ reducing $\mathrm{AgNO}_{3}$ on the surface of GO. The UV, XRD, Raman, TGA and TEM characterization all demonstrated the formation of GOA nanocomposites. Subsequently, GOA nanocomposite was dropped coating on sensor housing materials for the first time to study their surface and antifouling property. The surface study through AFM and WCA indicated the well distribution of Ag NPs in GOA nanocomposites induced homogeneous topology, high WCA and low SFE which in turn led to good antifouling property. By testing GO and GOA nanocomposites against $H$. Pacifica and a mixture of algae, we proved GOA1 possessed the best antifouling property. It showed biofilm inhibition of $83 \%$ against $H$. Pacifica and biofilm inhibition of $56 \%$ against a mixture of algae. Importantly, the antifouling property of GOA nanocomposite is found to depend on the Ag NPs' dispersibility. In conclusion, our results show that GOA nanocomposite possesses good antifouling property on senor housing materials, which indicates it might be a promising coating candidate in sensor antifouling field.

Acknowledgements The current work was sponsored by the Research Council of Norway throughout CtrlAQUA project [Grant Numbers: 237856], which is greatly acknowledged.

Open Access This article is licensed under a Creative Commons Attribution 4.0 International License, which permits use, sharing, adaptation, distribution and reproduction in any medium or format, as long as you give appropriate credit to the original author(s) and the source, provide a link to the Creative Commons licence, and indicate if changes were made. The images or other third party material in this article are included in the article's Creative Commons licence, unless indicated otherwise in a credit line to the material. If material is not included in the article's Creative Commons licence and your intended use is not permitted by statutory regulation or exceeds the permitted use, you will need to obtain permission directly from the copyright holder. To view a copy of this licence, visit http://creativecommons. org/licenses/by/4.0/. 


\section{References}

1. M. Cuartero, N. Pankratova, T. Cherubini, G. A. Crespo, F. Massa, F. Confalonieri, and E. Bakker (2017). Environ. Sci. Technol. Lett. 4, 410.

2. Z. A. Wang, F. N. Sonnichsen, A. M. Bradley, K. A. Hoering, T. M. Lanagan, S. N. Chu, T. R. Hammar, and R. Camilli (2015). Environ. Sci. Technol. 49, 4441.

3. T. Biard, L. Stemmann, M. Picheral, N. Mayot, P. Vandromme, H. Hauss, G. Gorsky, L. Guidi, R. Kiko, and F. Not (2016). Nature 532, 504.

4. L. Delauney, C. Compere, and M. Lehaitre (2010). Ocean Sci. 6, 503.

5. K. H. Chae, Y. M. Jang, Y. H. Kim, O. J. Sohn, and J. I. Rhee (2007). Sensor Actuat B Chem. 124, 153.

6. J. Chapman and F. Regan (2012). Adv. Eng. Mater. 14, B175.

7. L. Al-Naamani, S. Dobretsov, J. Dutta, and J. G. Burgess (2017). Chemosphere 168, 408.

8. D. M. Yebra, S. Kiil, and K. Dam-Johansen (2004). Prog. Org. Coat. 50, 75.

9. M. A. Champ (2000). Sci. Total Environ. 258, 21.

10. E. Ytreberg, J. Karlsson, and B. Eklund (2010). Sci. Total Environ. 408, 2459.

11. M. S. Selim, S. A. El-Safty, M. A. Shenashen, S. A. Higazy, and A. Elmarakbi (2020). J. Mater. Chem. B. 8, 3701.

12. M. S. Selim, S. A. El-Safty, N. A. Fatthallah, and M. A. Shenashen (2018). Prog. Org. Coat. 121, 160.

13. M. S. Selim, H. Yang, S. A. El-Safty, N. A. Fatthallah, M. A. Shenashen, F. Q. Wang, and Y. Huang (2019). Colloid Surf. A. 570, 518 .

14. M. S. Selim, S. A. El-Safty, A. M. Azzam, M. A. Shenashen, M. A. El-Sockary, and O. M. A. Elenien (2019). Chemistryselect. 4, 3395.

15. S. Chernousova and M. Epple (2013). Angew. Chem. Int. Edit. 52, 1636.

16. J. A. Lemire, J. J. Harrison, and R. J. Turner (2013). Nat. Rev. Microbiol. 11, 371.

17. N. Cioffi, L. Torsi, N. Ditaranto, G. Tantillo, L. Ghibelli, L. Sabbatini, T. Bleve-Zacheo, M. D'Alessio, P. G. Zambonin, and E. Traversa (2005). Chem. Mater. 17, 5255.

18. J. J. Harrison, H. Ceri, C. A. Stremick, and R. J. Turner (2004). Environ. Microbiol. 6, 1220.

19. C. N. Lok, C. M. Ho, R. Chen, Q. Y. He, W. Y. Yu, H. Z. Sun, P. K. H. Tam, J. F. Chiu, and C. M. Che (2006). J. Proteome Res. 5, 916.

20. Y. Yang and P. J. J. Alvarez (2015). Environ. Sci. Technol. Lett. 2, 221 .

21. J. R. Morones, J. L. Elechiguerra, A. Camacho, K. Holt, J. B. Kouri, J. T. Ramirez, and M. J. Yacaman (2005). Nanotechnology. 16, 2346.
22. S. Y. Lee, H. J. Kim, R. Patel, S. J. Im, J. H. Kim, and B. R. Min (2007). Polym. Adv. Technol. 18, 562.

23. K. D. Secinti, H. Ozalp, A. Attar, and M. F. Sargon (2011). J. Clin. Neurosci. 18, 391.

24. R. Wang, K. G. Neoh, E. T. Kang, P. A. Tambyah, and E. Chiong (2015). J. Biomed. Mater. Res. B. 103, 519.

25. J. Li and C. Y. Liu (2010). Eur. J. Inorg. Chem.. https://doi.org/ 10.1002/ejic.2009010481244.

26. C. N. R. Rao, A. K. Sood, K. S. Subrahmanyam, and A. Govindaraj (2009). Angew. Chem. Int. Edit. 48, 7752.

27. C. Soldano, A. Mahmood, and E. Dujardin (2010). Carbon 48, 2127.

28. G. Goncalves, P. A. A. P. Marques, C. M. Granadeiro, H. I. S. Nogueira, M. K. Singh, and J. Gracio (2009). Chem. Mater. 21, 4796.

29. J. Huang, L. M. Zhang, B. A. Chen, N. Ji, F. H. Chen, Y. Zhang, and Z. J. Zhang (2010). Nanoscale. 2, 2733.

30. R. Pasricha, S. Gupta, and A. K. Srivastava (2009). Small 5, 2253.

31. I. Ocsoy, M. L. Paret, M. A. Ocsoy, S. Kunwar, T. Chen, M. X. You, and W. H. Tan (2013). ACS Nano 7, 8972.

32. A. Soroush, W. Ma, Y. Silvino, and M. S. Rahaman (2015). Environ. Sci. Nano. 2, 395.

33. K. Krishnamoorthy, K. Jeyasubramanian, M. Premanathan, G. Subbiah, H. S. Shin, and S. J. Kim (2014). Carbon 72, 328.

34. S. Zinadini, A. A. Zinatizadeh, M. Rahimi, V. Vatanpour, and H. Zangeneh (2014). J. Membr. Sci. 453, 292.

35. M. S. L. Yee, P. S. Khiew, W. S. Chiu, Y. F. Tan, Y. Y. Kok, and C. O. Leong (2016). Colloid Surf. B. 148, 392.

36. M. D. Firouzjaei, A. A. Shamsabadi, S. A. Aktij, S. F. Seyedfour, M. Sharifian, A. Rahimpour, M. R. Esfahani, M. Ulbricht, and M. Soroush (2018). ACS Appl. Mater. Interface 10, 42967.

37. V. Vatanpour, A. Shockravi, H. Zarrabi, Z. Nikjavan, and A. Javadi (2015). J. Ind. Eng. Chem. 30, 342.

38. D. H. Kaelble (1970). J Adhesion. 2, 66.

39. D. K. Owens and R. C. Wendt (1969). J. Appl. Polym. Sci. 13, 1741.

40. J. Tang, Q. Chen, L. G. Xu, S. Zhang, L. Z. Feng, L. Cheng, H. $\mathrm{Xu}, \mathrm{Z}$. Liu, and R. Peng (2013). ACS Appl. Mater. Interface 5, 3867.

41. C. Deetuam, C. Samthong, S. Thongyai, P. Praserthdam, and A. Somwangthanaroj (2014). Compos. Sci. Technol. 105, 202.

42. W. Shao, X. F. Liu, H. H. Min, G. H. Dong, Q. Y. Feng, and S. L. Zuo (2015). ACS Appl. Mater. Interface 7, 6966.

43. J. P. Rourke, P. A. Pandey, J. J. Moore, M. Bates, I. A. Kinloch, R. J. Young, and N. R. Wilson (2011). Angew. Chem. Int. Edit. 50, 3173.

Publisher's Note Springer Nature remains neutral with regard to jurisdictional claims in published maps and institutional affiliations. 